\title{
ON THE REGULARITIES OF THE MEAN DISTANCES OF SECONDARY BODIES IN THE SOLAR SYSTEM
}

\author{
Z. ZAWISŁAWSKI ${ }^{1}$ W. KOSEK, ${ }^{2}$ and J. LELIWA-KOPYSTYNSKI ${ }^{3},{ }^{2}$ \\ ${ }^{1}$ Institute of Mathematics and Physics of Siedice University, \\ 08-110, Siedice, 3 Maja \\ 2 Space Research Center, PAS, 00-716 Warsaw, Bartycka 18A \\ ${ }^{3}$ Institute of Geophysics, Warsaw University, 02-093 Warsaw, Pasteura 7
}

(Received April 16, 1999)

\begin{abstract}
In this paper new formulae for the computation of orbital periods and mean distances of secondary bodies from a primary central body are given as a function (i) of an integer orbit number, and (ii) of the mass of the primary. Not all orbits numbered by the consecutive integers are occupied by the secondaries; on the contrary, most of them remain empty.

It has been found that the mass of the primary, for which the formulae are valid, must be greater than $\mathrm{Mo}=$ $1.747 \mathrm{M}_{\text {Earth }}$. The mean primary/secondary distances and orbital periods agree surprisingly accurately with the real ones for all systems considered (Solar, Jovian, Satumian, Uranian and Neptunian, Table 7).
\end{abstract}

KEY WORDS Solar system, planets, Titius-Bode rule

\section{INTRODUCTION}

The mean distances of planets from the Sun increase in some deterministic manner. They were first described by the Titius-Bode rule

$$
a_{k}=0.4+0.3 \times 2^{k}, \quad k=-\infty, 1,2, \ldots
$$

which is satisfied for all the planets except Neptune. But the Titius-Bode rule in its pristine form cannot be applied to the satellites of the planets. In the book of Nieto (1972) there is presented a comprehensive discussion of many generalizations of the Titius-Bode rule, including those valid for satellites systems. The generalizations:

(i) are based on simple fitting of multi-parameter mathematical formulae to the real distances (e.g. the Blagg-Richardson formulation), or (ii) they are supported by some physical premises (as in electromagnetic and gravitational theories), (iii) A more modern approach, mostly developed after the publication of Nieto's book, 
to an arrangement of primary/secondary mean distances must take into account the results of modelling of planet and satellite formation from primordial nebulae (e.g. see the papers of Cox and Lewis, 1980; Wetherill, 1980; Weidenschilling and Davis, 1985; Lissauer, 1995; and many others). However, in these works the central distances are rather the byproducts or even fixed parameters of an accretion theory to which a post-accretional system must converge. Alfven and Arrhenius (1976) simply rejected any physical significance of the Titius-Bode rule.

In short: the regularities concerning planetary and satellite distances exist but their origins are not clear as yet. The orbit/orbit and spin/orbit resonances are the only classes of exceptions, which can be explained on the basis of celestial mechanics. Since none of the calculations mentioned above are similar to our approach we therefore believe that our method is worth presenting. In this paper the regularities concerning mean distances and orbital periods are described on the basis of one assumption that the ratio of the distances of the allowed neighbour planets from the Sun is a constant value. A similar assumption concerns the satellites orbiting a giant planet. Hereafter, the Sun or the giant planets are called primary bodies. Corresponding to that the planets or the satellites of the giant planets are called the secondary bodies. It has been found that the mean distances from primary to secondary obey the rule, which is valid for five systems: the planetary system, and the four giant-planet satellite systems. Therefore our approach is much more general than that of the Titius-Bode rule. Contrary to the Titius-Bode rule our calculations show that only some of the 'allowed' mean distances and corresponding orbital periods are occupied by the secondary bodies. The results are in good agreement with observations.

\section{THE RELATIONS BETWEEN MEAN DISTANCES (OR ORBITAL PERIODS) OF THE SECONDARY BODIES IN THE SOLAR SYSTEM}

Let us assume that in the Solar System the ratio of the 'allowed' mean distances $a_{k}$ and $a_{k+1}$

from the primary body with the mass $M$ is a constant value:

$$
\frac{a_{k+1}}{a_{k}}=\gamma_{M}
$$

This formula presents only one, but essential, assumption of the paper.

From equation (2) it follows that the mean distance of a secondary body with number $k+n$ can be expressed by the mean distance with number $k$ by the formula:

$$
a_{k+n}=a_{k} \gamma_{M}^{n}
$$

From equation (3) and from Kepler's third law:

$$
\frac{T_{k}^{2}}{a_{k}^{3}}=\frac{T_{k+n}^{2}}{a_{k+n}^{3}}=\text { const. }
$$


it follows that the orbital period corresponding to the orbit with number $\mathrm{k}+\mathrm{n}$ is expressed by the formula:

$$
T_{k+n}=T_{k} \gamma_{M}^{3 n / 2}
$$

which is the crucial one for the further considerations concerning regularities between central distances in the planetary system as well as in satellite systems.

Let us describe in detail the calculations based on equation (5) applied to the planetary system. Similar calculations have been performed for the satellite systems of the giant planets.

Let us denote the orbital periods of Mercury, Venus, Earth, Mars, Jupiter, Saturn, Uranus, Neptune and Pluto as $\mathrm{T}_{k}, \mathrm{~T}_{k+l}, \quad \mathrm{~T}_{k+l+m}, \quad \mathrm{~T}_{k+l+m+n}, \quad \mathrm{~T}_{k+l+m+n+p}, \quad \mathrm{~T}_{k+l+m+n+p+q}, \quad \mathrm{~T}_{k+l+m+n+p+q+r}$, $\mathrm{T}_{k+l+m+n+p+q+r+s}$ and $\mathrm{T}_{k+l+m+n+p+q+r+s+t}$, respectively. Here $k$ is an optional integer and the other integers $l, m, n, p, q, r, s, t$ are positive. After dividing equation (5) by $\mathrm{T}_{k}$ and taking logarithms of both sides we get:

$$
\begin{gathered}
\log \left(\frac{T_{k+l}}{T_{k}}\right)=l \beta_{M} \\
\log \left(\frac{T_{k+l+m}}{T_{k}}\right)=(l+m) \beta_{M} \\
\log \left(\frac{T_{k+l+m+\ldots}}{T_{k}}\right)=(l+m+\ldots) \beta_{M} \\
\log \left(\frac{T_{k+l+m++n+p+q+r+s+t}}{T_{k}}\right)=(l+m++n+p+q+r+s+t) \beta_{M} \\
\quad \beta_{M}=3 / 2 \log \left(\gamma_{M}\right)
\end{gathered}
$$

In the eight equations (6.1)-(6.8) there are nine unknowns, namely eight positive integers $l, m$, $n, p, q, r, s, t$ and a positive real $\beta_{M}$ - To determine all nine unknowns it is necessary to assume one of them is a parameter. We are looking for the smallest values of the unknown integers, thus it is convenient to assume one (for example $l$ ) of them as a parameter. If we start our calculations from $l=1$ then $\beta_{M}$ is simply given by equation (6.1); next, from equation (6.2) with the previously found $\beta_{M}$ we can derive $m$ as the closest integer number to the computed real value of $m$. In the following step, we update the new value for $\beta_{M}$ as the mean from equations (6.1) and (6.2) taking the previously calculated integers $l$ and $m$. Next, the third unknown integer $n$ can be determined from equation (6.3), etc. If these calculations give one of the consecutive unknown integers equal to zero then we must reject the previous results and start the calculations from the beginning with the new value $l=2$. If $l=2$ does not satisfy the condition that the consecutive unknown integers are positive we must repeat the calculations with $l=3$, etc. Indeed, in the case of the Sun and nine planets we have found that $l=1$ does not satisfy the required conditions; however the next value $l=2$ allows us to solve the set of equations (6) leading to positive integers: $m=1, n=1$, $p=4, q=2, r=2, s=1$ and $t=1$. 
Table 1. The coefficients $\beta_{M}$ and their standard errors for the Sun, Jupiter, Saturn, Uranus and Neptune computed from orbital periods of their secondary bodies.

\begin{tabular}{|l|l|l|l|}
\hline $\begin{array}{l}\text { Central } \\
\text { body }\end{array}$ & $\begin{array}{l}\text { Number of secondary } \\
\text { bodies (N) }\end{array}$ & $\begin{array}{l}\text { Number of pair of secondary } \\
\text { bodies (N / 2) }\end{array}$ & $\beta_{M}$ (robust mean) \\
\hline Sun & 9 & 36 & $0.2159 \pm 0.0117$ \\
Jupiter & 7 & 21 & $0.0907 \pm 0.0054$ \\
Saturn & 10 & 45 & $0.0710 \pm 0.0020$ \\
Uranus & 6 & 15 & $0.0378 \pm 0.0010$ \\
Neptune & 6 & 15 & $0.0405 \pm 0.0011$ \\
\hline
\end{tabular}

From this moment the integers $l, m, \ldots, t$ are treated as known values. However, the value of $\beta_{M}$ computed in the last step as well as in the previous steps does not satisfy all of these equations simultaneously. Therefore $\beta_{M}$ must be adjusted using an appropriate mathematical method. So it was computed as the robust mean (Press et al., 1992) using all the combinations of orbital periods of pairs of planets from all the planets. The robust estimate enables the elimination of big errors in $\beta_{M}$ these errors are caused by the fact that one of the planets is not on the orbit corresponding to an integer number. The final result for $\beta_{M}$ for the planetary system is shown in the first row of Table 1. Using the same algorithm the integer numbers $l, m, n, p, \ldots$ and the mean values of $\beta_{M}$ were computed for the satellite systems of Jupiter, Saturn and Uranus (Table 1, next three rows). It can be seen that the calculated $\beta_{M}$ for four different primary-secondary systems are in very good correlation with the logarithm of the central body mass M (Figure 1).

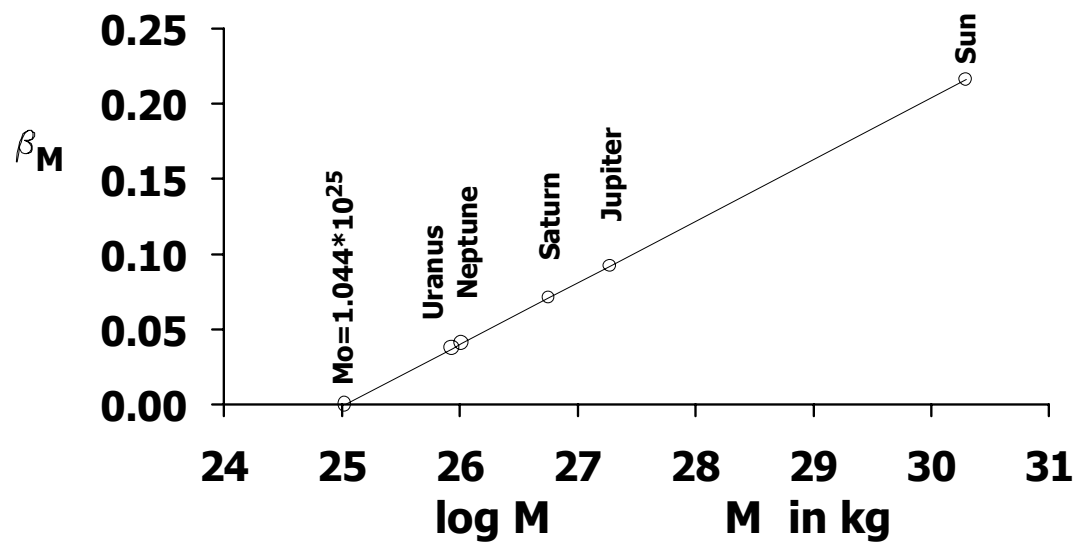

Figure 1. The $\beta_{M}$ computed by the robust method from orbital periods of the secondary bodies of the Sun, Jupiter, Saturn, Uranus andNeptune as a function of the central body mass M (in kilograms).

$\beta_{M}=3 / 2$ z $\log (\mathrm{M} / \mathrm{Mo})$, where $\mathrm{z}=0.02725$ and $\mathrm{Mo}=1.044 \times 10^{25} \mathrm{~kg}=1.747 \mathrm{M}_{\text {Earth }}$. 
Table 2. The observed and computed orbital periods and mean distances of planets and their comparison with TitiusBode mean distances. In this table, as well as in Tables 3-6, and in Figures 2-6, the orbital number equal to zero is assigned to the reference secondary body of the considered system; each time it is a secondary with the largest mass.

\begin{tabular}{|c|c|c|c|c|c|c|}
\hline Planet & $\begin{array}{l}\text { Orbit } \\
\text { number }\end{array}$ & $\mathbf{T}_{\text {comp }}$ & $\mathbf{T}_{\text {real }}$ & $\mathbf{a}_{\text {comp }}$ & $\mathbf{a}_{\text {real }}$ & $\begin{array}{l}\mathbf{a}_{\text {comp }} \\
\text { Titius-Bode }\end{array}$ \\
\hline & & (years) & (years) & (a.u.) & (a.u.) & (a.u.) \\
\hline & -9 & 0.136 & & 0.264 & & \\
\hline \multirow[t]{2}{*}{ Mercury } & -8 & 0.223 & 0.241 & 0.368 & 0.387 & 0.4 \\
\hline & -7 & 0.366 & & 0.512 & & \\
\hline Venus & -6 & 0.602 & 0.615 & 0.713 & 0.712 & 0.7 \\
\hline Earth & -5 & 0.989 & 1.000 & 0.993 & 1.000 & 1.0 \\
\hline Mars & -4 & 1.626 & 1.880 & 1.383 & 1.524 & 1.6 \\
\hline Hungaria* & -3 & 2.672 & $2.6^{*}$ & 1.926 & $1.9 *$ & \\
\hline Asteroids** & -2 & 4.391 & $4.28 * *$ & 2.683 & $2.636 * *$ & 2.8 \\
\hline- & -1 & 7.217 & & 3.738 & & \\
\hline \multirow[t]{2}{*}{ Jupiter } & $\mathbf{0}$ & 11.862 & 11.862 & 5.203 & 5.203 & 5.2 \\
\hline & 1 & 19.50 & & 7.246 & & \\
\hline Saturn & 2 & 32.04 & 29.46 & 10.09 & 9.537 & 10.0 \\
\hline Chiron*** & 3 & 52.66 & $50.7 * * *$ & 14.05 & $13.7 * * *$ & \\
\hline Uranus & 4 & 86.55 & 84.01 & 19.57 & 19.18 & 19.6 \\
\hline Neptune & 5 & 142.3 & 164.8 & 27.26 & 30.06 & \\
\hline \multirow[t]{4}{*}{ Pluto } & 6 & 233.8 & 247.7 & 37.97 & 39.44 & 38.8 \\
\hline & 7 & 384.3 & & 52.88 & & \\
\hline & 8 & 631.6 & & 73.64 & & 77.2 \\
\hline & 9 & 1038.0 & & 102.6 & & \\
\hline
\end{tabular}

*Hungaria group of asteroids, according to Alfven and Arrhenius (1976).

**The 'asteroids' orbital period Treal and mean solar distance areal are artificial, since they denote the geometrical averages for four asteroids, namely 1 Ceres, 2 Pallas, 3 Juno and 4 Vesta. This choice of asteroids follows that of Nieto (1972) and corresponds to some of the largest and most massive asteroids. Indeed, according to Tedesco et al. (1989) the diameters of 1 Ceres, 2 Pallas, 4 Vesta, and 3 Juno are $913 \mathrm{~km}, 523 \mathrm{~km}, 501 \mathrm{~km}$, and $244 \mathrm{~km}$, respectively; according to Millis and Dunhem (1989) the masses of 1 Ceres, 2 Pallas, 4 Vesta are $(5.9 \pm 0.3),(1.08 \pm 0.22)$ and $(1.38 \pm$ 0.12 ) in units of $10^{-10} \mathrm{M}_{\text {Sun }}$.

***Chiron data, according to French et al. (1989), are $\mathrm{T}=50.7 \mathrm{y}, \mathrm{a}=13.7$ a.u., and eccentricity $\mathrm{e}=0.38$. The Chiron radius, according to Stern (1994), is in the range 83-150 km. Comparison of real Chiron parameters $T$, $a$ with our calculated ones presents surprisingly good agreement. However, since Chiron's orbit is chaotic and subject to strong perturbations due to Saturn, there are two possibilities of an interpretation of this agreement: (i) it is rather accidental, or (ii) Chiron's orbital period and mean solar distance are 'as they are', because they are oscillating around the parameters predicted by our method. 


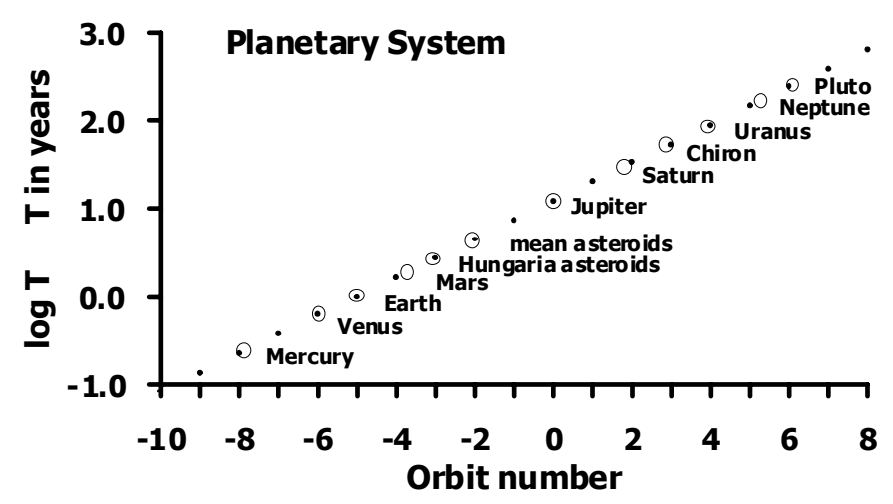

Figure 2. The logarithm of the computed (dots) and observed (circles) orbital periods of the planets as a function of the orbit number. The 'mean asteroids', the Hungaria group of asteroids, and Chiron were not taken into account in the calculations by means of equations (6.1)-(6.8). Their 'real orbit numbers' were calculated to put them on a straight line, which is based on calculations for the nine planets.

procedure for $l, m, n, p, \ldots$ calculations cannot be applied to the satellites of Neptune since the number of moons is very low comparing with the number of expected positions (Figure 6). The value of $\beta_{M}$ for Neptune, in the first approximation, has been interpolated on the basis of its known mass put on the regression line (Figure 1 and Table 1 the bottom row). Next, for this known Neptunian parameter $\beta_{M}$ the values of $l, m, n, p, \ldots$, and the final value of $\beta_{M}$ were calculated.

The correlation coefficient between $\beta_{M}$ computed as the robust mean and $\log (\mathrm{M})$ is equal to 0.99994. When $\beta_{M}$ were computed as the mean arithmetic value then the correlation coefficient is smaller and equal to 0.99991 . These correlation coefficients are significant at the $99.0 \%$ confidence level. From Figure 1 and the high correlation coefficients it can be seen that $\beta_{M}$ is a straight line function of $\log (\mathrm{M})$ given by the formula:

$$
\beta_{M}=\frac{3}{2} z \log (M)-\frac{3}{2} z \log \left(M_{o}\right)
$$

where $z$ and Mo are constants to be found. Taking into account equation (7) we find that this value of $\beta_{M}$ gives $\gamma_{M}=1$; therefore this means that our fundamental starting equation (2) loses its sense if the primary body mass is less than Mo. In practice, this means that we cannot apply our method to deduce regularities in primary/secondary distances in low-mass and few-member systems (e.g. Earth, Mars, Pluto, and the asteroid Ida).

When the pair $\beta_{M}$ and $\mathrm{M}$ in equation (8) are replaced by the pairs $\beta_{S u n}, M_{S u n}$; $\beta_{\text {Jupiter }}, M_{\text {Jupiter }} ; \beta_{\text {Saturn }}, M_{\text {Saturn }} ; \beta_{\text {Uranus }}, M_{\text {Uranus }}$ and $\beta_{\text {Neptune }} M_{\text {Nepune }}$ then five independent equations of the type of equation (8) can be created. Therefore, we have obtained a system of five equations with two unknowns $z$ and Mo. They were determined using a robust straight-line fit (Press et al., 1992) through a set of data points: $z=0.02725$ and Mo $=1.044 \mathrm{x}$ $10^{25} \mathrm{~kg}=1.747 \mathrm{M}_{\text {Earth. }}$

Comparison of the right-hand side of equations (7) and (8) shows that $\beta_{M}$ is a 
Table 3. The observed and computed orbital periods and the mean distances of the Jovian satellites.

\begin{tabular}{|c|c|c|c|c|c|}
\hline Satellite & Orbit number & $\begin{array}{l}\mathbf{T}_{\text {comp }} \\
\text { (days) }\end{array}$ & $\begin{array}{r}\mathbf{T}_{\text {real }} \\
\text { (days) }\end{array}$ & $\begin{array}{c}\mathbf{a}_{\text {comp }} \\
\left(10^{6} \mathrm{~km}\right)\end{array}$ & $\begin{array}{r}\mathbf{a}_{\text {real }} \\
\left(10^{6} \mathrm{~km}\right)\end{array}$ \\
\hline & -16 & 0.238 & & 0.111 & \\
\hline \multirow[t]{2}{*}{ Adrastea } & -15 & 0.295 & 0.2983 & 0.128 & 0.1290 \\
\hline & -14 & 0.364 & & 0.147 & \\
\hline \multirow[t]{2}{*}{ Amalthea } & -13 & 0.451 & 0.489 & 0.169 & 0.1813 \\
\hline & -12 & 0.558 & & 0.195 & \\
\hline \multirow[t]{3}{*}{ Thebe } & -11 & 0.690 & 0.675 & 0.225 & 0.2219 \\
\hline & -10 & 0.853 & & 0.259 & \\
\hline & -8 & 1.305 & & 0.344 & \\
\hline \multirow[t]{3}{*}{ Lo } & -7 & 1.615 & 1.769 & 0.397 & 0.4216 \\
\hline & -6 & 1.997 & & 0.457 & \\
\hline & -4 & 3.056 & & 0.607 & \\
\hline \multirow[t]{3}{*}{ Europa } & -3 & 3.780 & 3.551 & 0.699 & 0.6709 \\
\hline & -2 & 4.676 & & 0.806 & \\
\hline & -1 & 5.784 & & 0.929 & \\
\hline \multirow[t]{3}{*}{ Ganymede } & 0 & 7.155 & 7.155 & 1.070 & 1.070 \\
\hline & 1 & 8.851 & & 1.233 & \\
\hline & 3 & 13.54 & & 1.637 & \\
\hline \multirow[t]{2}{*}{ Callisto } & 4 & 16.75 & 16.689 & 1.887 & 1.883 \\
\hline & 5 & 20.72 & & 2.174 & \\
\hline
\end{tabular}

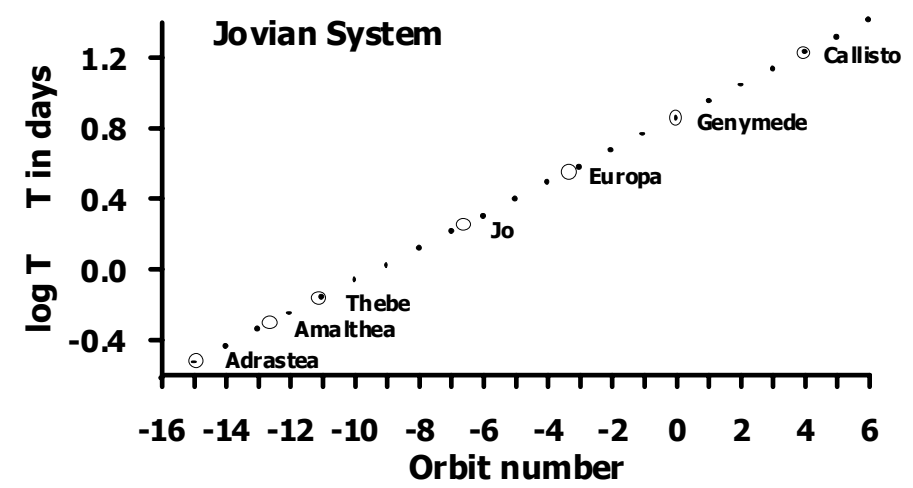

Figure 3. The logarithm of the computed (dots) and observed (circles) orbital periods of the Jovian satellites as a function of the orbit number 
function of the central body mass M:

$$
\frac{3}{2} \log \left(\gamma_{M}\right)=\frac{3}{2} z \log \frac{M}{M_{o}} \rightarrow \gamma_{M}=\left(\frac{M}{M_{o}}\right)^{z}
$$

Substituting $\gamma_{M}$ expressed by formula (9) into equations (3) and (5), the orbital period and mean distance of a secondary body with number $k+n$ can be determined from the orbital period and mean distance with number $k$ using the recursion formulae:

$$
\begin{aligned}
& a_{k+n}=a_{k}\left(\frac{M}{M_{o}}\right)^{z n} \\
& T_{k+n}=T_{k}\left(\frac{M}{M_{o}}\right)^{\frac{3}{2} z n}
\end{aligned}
$$

The orbit number $k=0$ in equations (10) and (11) can be assigned for any secondary body arbitrarily chosen. In this paper the orbit number 0 was chosen for the secondary body having the largest mass. They are Jupiter, Ganymede, Titan, Titania and Triton for the Solar, Jovian, Saturnian, Uranian and Neptunian systems, respectively. The observed and computed orbital periods and mean distances of secondary bodies are listed in Tables 2-6. The logarithms of the computed and observed orbital periods of the secondary bodies are shown in Figures 2-6. The integer numbers are related to the computed orbital periods and mean distances. The numerical data for Tables 2-6 as well as for Figures 2-6 are $z=0.027246$ and Mo $=1.0438 \times 10^{25} \mathrm{~kg}=$ 1.747M $\mathrm{M}_{\text {Earth. }}$

\section{STATISTICAL VERIFICATION OF THE RESULTS}

From Figures 2-6 we can see that the calculated positions (described by integer numbers) of secondary bodies differ slightly from the real ones. Therefore, to verify the results some statistical analysis is necessary. To each observed orbital period of a secondary body a real orbital number can be attributed. This real number differs from a neighbouring integer by no more than 0.5 . A simple statistical algorithm described below takes into account the absolute values of these differences.

Equation (11) is a linear relation between orbital numbers and logarithms of orbital periods. Therefore, the difference between the logarithms of the computed and the nearest observed orbital period divided by the difference of the logarithms of neighbouring computed orbital periods is the absolute value of the difference between the real (in reality) and integer (in the model) value of an orbital number:

$$
\varepsilon_{k}=\frac{\min \left[\log \left(T / T_{k}\right), \log \left(T_{k+1} / T\right)\right]}{\log \left(T_{k+1} / T_{k}\right)}
$$

Here $\mathrm{T}, \mathrm{T}_{k}<\mathrm{T}<\mathrm{T}_{k+1}$, is a real orbital period of a secondary body. According to this definition $0<\mathcal{E}_{k}<0.5$. 
Table 4. The observed and computed orbital periods and the mean distances of the Saturnian satellites.

\begin{tabular}{|c|c|c|c|c|c|}
\hline Satellite & Orbit number & $\begin{array}{c}\mathbf{T}_{\text {comp }} \\
\text { (days) }\end{array}$ & $\begin{array}{r}\mathbf{T}_{\text {real }} \\
\text { (days) }\end{array}$ & $\begin{array}{c}\mathbf{a}_{\text {comp }} \\
\left(10^{6} \mathrm{~km}\right)\end{array}$ & $\begin{array}{c}a_{\text {real }} \\
\left(10^{6} \mathrm{~km}\right)\end{array}$ \\
\hline & -20 & 0.608 & & 0.138 & \\
\hline \multirow[t]{2}{*}{ Janus* } & -19 & 0.715 & 0.695 & 0.154 & 0.1514 \\
\hline & -18 & 0.842 & & 0.172 & \\
\hline \multirow[t]{2}{*}{ Mimas } & -17 & 0.992 & 0.942 & 0.192 & 0.1855 \\
\hline & -16 & 1.168 & & 0.214 & \\
\hline \multirow[t]{2}{*}{ Enceladus } & -15 & 1.375 & 1.370 & 0.239 & 0.2380 \\
\hline & -14 & 1.619 & & 0.266 & \\
\hline \multirow[t]{2}{*}{ Tethys } & -13 & 1.906 & 1.888 & 0.297 & 0.2947 \\
\hline & -12 & 2.245 & & 0.331 & \\
\hline \multirow[t]{3}{*}{ Dione } & -11 & 2.643 & 2.737 & 0.369 & 0.3774 \\
\hline & -10 & 3.112 & & 0.411 & \\
\hline & -9 & 3.665 & & 0.459 & \\
\hline \multirow[t]{3}{*}{ Rhea } & -8 & 4.315 & 4.518 & 0.511 & 0.5271 \\
\hline & -7 & 5.081 & & 0.570 & \\
\hline & -1 & 13.54 & & 1.096 & \\
\hline \multirow[t]{2}{*}{ Titan } & 0 & 15.945 & 15.945 & 1.222 & 1.222 \\
\hline & 1 & 18.78 & & 1.363 & \\
\hline \multirow[t]{3}{*}{ Hiperion } & 2 & 22.11 & 21.23 & 1.519 & 1.481 \\
\hline & 3 & 26.03 & & 1.694 & \\
\hline & 9 & 69.38 & & 3.257 & \\
\hline \multirow[t]{3}{*}{ lapetus } & 10 & 81.69 & 79.33 & 3.632 & 3.561 \\
\hline & 11 & 96.19 & & 4.050 & \\
\hline & 21 & 492.8 & & 12.04 & \\
\hline \multirow[t]{2}{*}{ Phoebe } & 22 & 580.3 & 550.4 & 13.42 & 12.95 \\
\hline & 23 & 683.3 & & 14.96 & \\
\hline
\end{tabular}

*Denote the coorbital satellites Janus and Epimatheus.

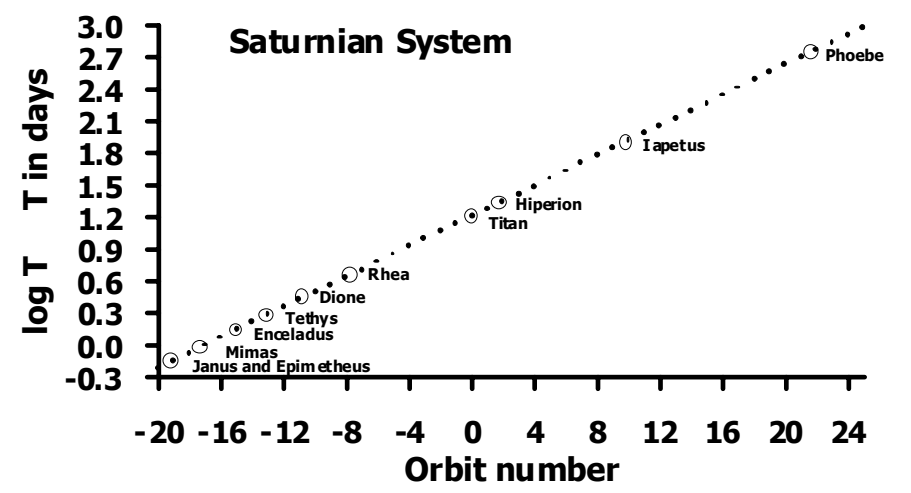

Figure 4. The logarithm of the computed (dots) and observed (circles) orbital periods of the Saturnian satellites as a function of the orbit number. 
Table 5. The observed and computed orbital periods and the mean distances of the Uranian satellites.

\begin{tabular}{|c|c|c|c|c|c|}
\hline Satellite & Orbit number & $\begin{array}{l}\mathrm{T}_{\text {comp }} \\
\text { (days) }\end{array}$ & $\begin{array}{r}T_{\text {real }} \\
\text { (days) }\end{array}$ & $\begin{array}{c}\mathbf{a}_{\text {comp }} \\
\left(10^{6} \mathrm{~km}\right)\end{array}$ & $\begin{array}{c}a_{\text {real }} \\
\left(10^{6} \mathrm{~km}\right)\end{array}$ \\
\hline & -29 & 0.707 & & 0.0817 & \\
\hline \multirow[t]{3}{*}{$1985 \mathrm{U} 1 *$} & -28 & 0.771 & $0.7625 *$ & 0.0866 & $0.0860 *$ \\
\hline & -27 & 0.841 & & 0.0917 & \\
\hline & -22 & 1.300 & & 0.122 & \\
\hline \multirow[t]{3}{*}{ Miranda } & -21 & 1.413 & 1.413 & 0.130 & 0.1298 \\
\hline & -20 & 1.541 & & 0.137 & \\
\hline & -15 & 2.376 & & 0.183 & \\
\hline \multirow[t]{3}{*}{ Ariel } & -14 & 2.591 & 2.52 & 0.194 & 0.1912 \\
\hline & -13 & 2.825 & & 0.206 & \\
\hline & -10 & 3.663 & & 0.245 & \\
\hline \multirow[t]{3}{*}{ Umbriel } & -9 & 3.994 & 4.144 & 0.259 & 0.2660 \\
\hline & -8 & 4.355 & & 0.275 & \\
\hline & -1 & 7.984 & & 0.411 & \\
\hline \multirow[t]{3}{*}{ Titania } & 0 & 8.706 & 8.706 & 0.4358 & 0.4358 \\
\hline & 1 & 9.493 & & 0.462 & \\
\hline & 4 & 12.31 & & 0.549 & \\
\hline \multirow[t]{2}{*}{ Oberon } & 5 & 13.42 & 13.46 & 0.582 & 0.5826 \\
\hline & 6 & 14.64 & & 0.616 & \\
\hline
\end{tabular}

*Orbital period, central distance, and measured radius $(77 \pm 3) \mathrm{km}$ of satellite 1985U1 Puck are according to Veverka (1991).

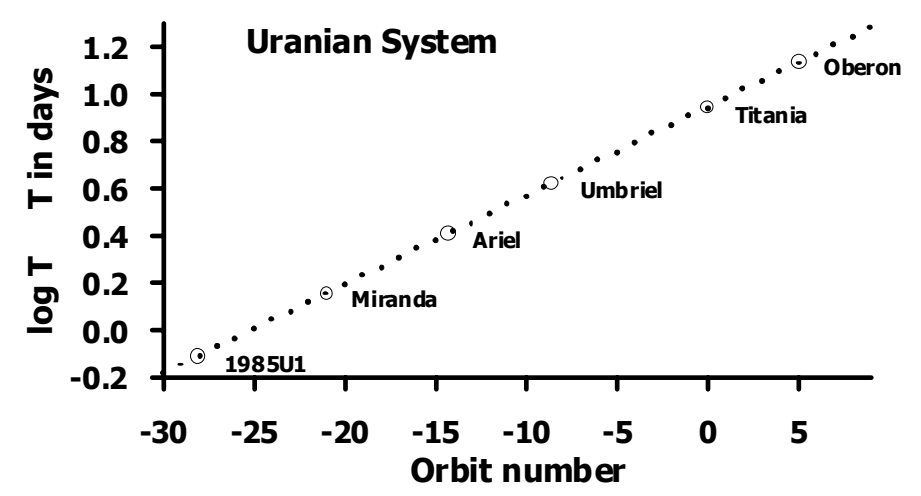

Figure 5. The logarithm of the computed (dots) and observed (circles) orbital periods of the Uranian satellites as a function of the orbit number. 
Table 6. The observed and computed orbital periods and the mean distances of the Neptunian satellites.

\begin{tabular}{|c|c|c|c|c|c|}
\hline Satellite & Orbit number & $\begin{array}{l}\mathrm{T}_{\text {comp }} \\
\text { (days) }\end{array}$ & $\begin{array}{r}T_{\text {real }} \\
\text { (days) }\end{array}$ & $\begin{array}{c}\mathbf{a}_{\text {comp }} \\
\left(10^{6} \mathrm{~km}\right)\end{array}$ & $\begin{array}{c}\mathbf{a}_{\text {real }} \\
\left(10^{6} \mathrm{~km}\right)\end{array}$ \\
\hline & -32 & 0.296 & & 0.0484 & \\
\hline \multirow[t]{3}{*}{ 1989N3 } & -31 & 0.325 & 0.333 & 0.0515 & 0.0525 \\
\hline & -30 & 0.357 & & 0.0549 & \\
\hline & -29 & 0.392 & & 0.0584 & \\
\hline \multirow[t]{3}{*}{ 1989N4 } & -28 & 0.431 & 0.429 & 0.0621 & 0.0620 \\
\hline & -27 & 0.473 & & 0.0661 & \\
\hline & -26 & 0.519 & & 0.0704 & \\
\hline \multirow[t]{3}{*}{$1989 \mathrm{~N} 2$} & -25 & 0.570 & 0.554 & 0.0749 & 0.0736 \\
\hline & -24 & 0.625 & & 0.0797 & \\
\hline & -19 & 0.997 & & 0.109 & \\
\hline \multirow[t]{3}{*}{ 1989N1 } & -18 & 1.095 & 1.121 & 0.116 & 0.1176 \\
\hline & -17 & 1.202 & & 0.123 & \\
\hline & -1 & 5.351 & & 0.333 & \\
\hline \multirow[t]{3}{*}{ Triton } & 0 & 5.875 & 5.875 & 0.3548 & 0.3548 \\
\hline & 1 & 6.450 & & 0.378 & \\
\hline & 43 & 325.2 & & 5.153 & \\
\hline \multirow[t]{2}{*}{ Nereid } & 44 & 357.0 & 360.1 & 5.484 & 5.513 \\
\hline & 45 & 391.9 & & 5.836 & \\
\hline
\end{tabular}

*Orbital periods, central distances, and radii 90, 75, 95, 200, 1352, and $170 \mathrm{~km}$ for satellites N3, N4, N2, N1, Triton and Nereid, respectively, are according to Stone and Miner (1989).

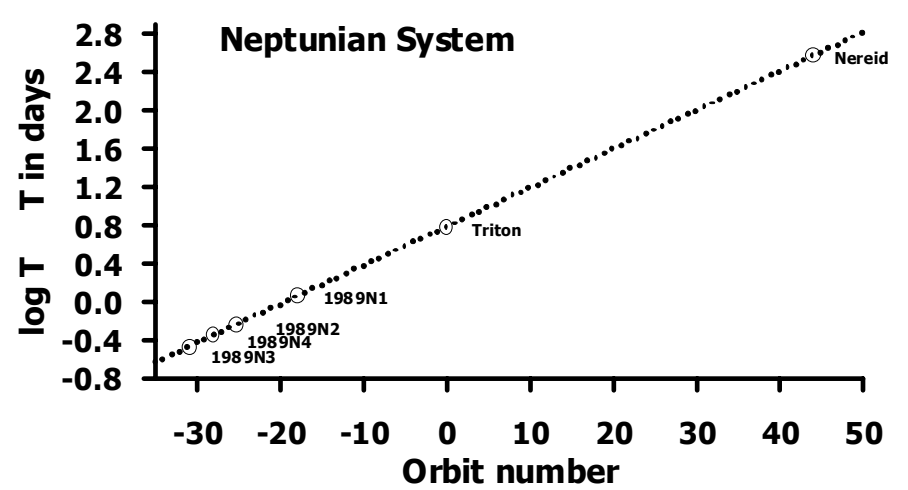

Figure 6. The logarithm of the computed (dots) and observed (circles) orbital periods of the Neptunian satellites as a function of the orbit number. 
Table 7. The computed mean value of errors $\langle\varepsilon\rangle$, equation (13), of the orbit numbers of the primary body systems.

\begin{tabular}{|c|c|c|c|c|c|}
\hline \multirow[t]{2}{*}{ No } & \multirow[t]{2}{*}{ System } & \multirow{2}{*}{$\begin{array}{l}\text { Number of } \\
\text { secondary } \\
\text { bodies }\end{array}$} & \multicolumn{3}{|c|}{$<\varepsilon>$} \\
\hline & & & This paper & Titius-Bode & \\
\hline $1 \mathrm{a}$ & planets, Neptune excluded planets, & 8 & 0.108 & 0.043 & \\
\hline $1 b$ & Nepune excluded + averge asteroids & 9 & 0.102 & 0.050 & \\
\hline $1 \mathrm{c}$ & $\begin{array}{l}\text { all planets } \\
\text { planets }+ \text { average asteroids }\end{array}$ & 9 & 0.129 & - & - \\
\hline $1 d$ & Jovian Satellites $\quad$ Saturnian & 10 & 0.121 & - & - \\
\hline 2 & Uranian Satellites & 7 & 0.183 & - & \\
\hline 3 & Neptunian satellites & 10 & 0.182 & - & \\
\hline 4 & average value* for all secondary bodies & 6 & 0.150 & & \\
\hline 5 & of the five considered systems & 6 & 0.155 & - & \\
\hline & $10+2+5+4+5$ & 39 & $0.157 \pm 0.025$ & & \\
\hline
\end{tabular}

Note: The tests of accuracy of our results are based on formulae (12) and (13). Four modifications of an approach to a planetary system (la-Id) and four satellite systems (2-5) were considered. For all the systems $\langle\varepsilon\rangle$ are considerably less than $E[<\varepsilon>]=0.25$ which corresponds to random numbers with a uniform distribution. For comparison, similar calculations of $\langle\varepsilon\rangle$ for the Titius-Bode rule applied to a planetary system have been performed. In the case of a planetary system our method is less accurate than the Titius-Bode rule. However, in contrast to the Titius-Bode rule, it is valid for diverse subsystems of the Solar System.

"The mean value of 39 random numbers with a uniform distribution is about 0.25 with standard deviation 0.04 . So, our result $0.157 \pm 0.025$ is considerably outside the range for random numbers.

The accuracy of a model can be measured by calculating the mean value of all of the epsilons (the mean value of the error of 'the real orbit number')

$$
<\varepsilon>=\frac{1}{N} \sum \varepsilon_{k}
$$

where $N$ is the total number of secondary bodies in the system, see Table 7. If $\varepsilon_{k}$ of the secondary bodies were random numbers with a uniform distribution ranging from 0 to 0.5 then the expected mean value of them would be $E[<\varepsilon>]=0.25$. We can see in the case of all the systems considered that the value of $\langle\varepsilon\rangle$ are considerably less than 0.25 (Table 7). This presents a strong argument that our values of logarithms of orbital periods of secondary bodies are not uniformly distributed random numbers. Thus, orbital periods and mean distances are not random either. Similar statistics has been performed for the mean distances of eight planets as well as eight planets plus an 'average asteroid' computed using the formulae of this paper and the Titius-Bode rule (Table 7); the Titius-Bode rule does not hold for Neptune at all. 
The orbital periods of secondary bodies exceed the theoretical ones probably due to mutual gravitational attractions of these secondary bodies. If these mutual gravitational attractions were taken into account then the orbital period errors would probably be smaller, and this needs further investigations.

\section{CONCLUSIONS}

The results of this paper are based only on one simple assumption (2) that the ratio of mean distances attributed to the 'allowed' orbits of secondary bodies is a constant value for the considered primary/secondaries system. This constant value depends only on the mass of the primary body of the system. It has been found that our calculation method of central distances of secondaries can be valid for such systems in which the mass of the primary body exceeds Mo = $1.747 \mathrm{M}_{\text {Earth- }}$ Although this mass has been found with quite good accuracy, we cannot at present give a physical interpretation of Mo. The statistical analysis of the results shows rather clearly that the five subsystems of our Solar System obey the initial assumption and the orbits of secondaries are not randomly distributed. The calculations also reveal such mean distances or orbital periods corresponding to them, which are not occupied by planets or satellites. The orbits with mean solar distances of 73.64 and 102.6 a.u. (Table 2) represent the hypothetical Kuiper belt.

\section{Acknowledgements}

The first author would like to thank Prof. W. Zych from the Technical University of Warsaw for his valuable comments and discussions.

\section{References}

Alfven, H. and Arrhenius, G. (1976) Evolution of the Solar System, NASA SP-345, Washington,

D.C.

Cox, L. P. and Lewis, J. S. (1980) Icarus 44, 706.

French, L. M., Vilas, F., Hartmann, W. K., and Tholen D. J. (1989) In. R.P. Binzel, T. Gehrels and M. S. Matthews (eds.) Asteroids II, pp. 468-486, University of Arizona Press, Tucson.

Lissauer, J. J. (1995) Icarus 114, 217.

Millis R. L. and Dunham D. W. (1989) In: R. P. Binzel, T. Gehrels and M. S. Matthews (eds.) Asteroids //, pp. 148-170, University of Arizona Press, Tucson.

Nieto, M. N. (1972) The Titius-Bode Law of Planetary Distances: Its History and Theory, Pergamon Press, Oxford.

Press, W. H., Flannery, B. P., Teukolsky, S. A., and VetterUng, W. T. (1992) Numerical Recipes, Cambridge University Press, Cambridge.

Stem, A. (1994) Bulletin of AAS 26, No. 3, 1152.

Stone, E. C. and Minar E. D (1989) Science 246, 1417.

Tedesco, E. F., Wiliams, J. G., Matson, D. L., Veeder, G. J., Gradie, J. C., and Lebofsky, L. A. (1989) In: R. P. Binzel, T. Gehrels and M. S. Matthews (eds.) Asteroids //, pp. 1151-1161, University of Arizona Press, Tucson.

Veverka, J., Brown, R. H., and Bell J. F. (1991) In: J. T. Bergstralh, E. D. Miner, and M. S. Matthews (eds.) Uranus, pp. 528-560, University of Arizona Press, Tucson. 
Wetherill, G. W. (1980) Ann. Rev. Astron. Astrophys. 18, 77.

Weidenschilling, S. J. and Davis, D. R. (1985) /carus 62, 16. 